\title{
Relative acceleration noise mitigation for nanocrystal matter-wave interferometry: Applications to entangling masses via quantum gravity
}

\author{
Marko Toroš $\odot,{ }^{1,2}$ Thomas W. van de Kamp $\odot,{ }^{3}$ Ryan J. Marshman, ${ }^{1}$ M. S. Kim ${ }^{4}$ Anupam Mazumdar, ${ }^{3,5}$ and Sougato Bose ${ }^{1, *}$ \\ ${ }^{1}$ University College London, Gower Street, WC1E 6BT London, United Kingdom \\ ${ }^{2}$ School of Physics and Astronomy, University of Glasgow, G12 8QQ Glasgow, United Kingdom \\ ${ }^{3}$ University of Groningen, PO Box 72, 9700 Groningen, Netherlands \\ ${ }^{4}$ QOLS, Blackett Laboratory, Imperial College London, SW7 2AZ London, United Kingdom \\ ${ }^{5}$ Van Swinderen Institute, University of Groningen, 9747 AG Groningen, Netherlands
}

(Received 22 August 2020; accepted 12 April 2021; published 4 June 2021)

\begin{abstract}
Matter-wave interferometers with large momentum transfers, irrespective of specific implementations, will face a universal dephasing due to relative accelerations between the interferometric mass and the associated apparatus. Here we propose a solution that works even without actively tracking the relative accelerations: putting both the interfering mass and its associated apparatus in a freely falling capsule, so that the strongest inertial noise components vanish due to the equivalence principle. In this setting, we investigate two of the most important remaining noise sources: (a) the noninertial jitter of the experimental setup and (b) the gravity-gradient noise. We show that the former can be reduced below desired values by appropriate pressures and temperatures, while the latter can be fully mitigated in a controlled environment. We finally apply the analysis to a recent proposal for testing the quantum nature of gravity [S. Bose et al., Phys. Rev. Lett. 119, 240401 (2017)] through the entanglement of two masses undergoing interferometry. We show that the relevant entanglement witnessing is feasible with achievable levels of relative acceleration noise.
\end{abstract}

DOI: 10.1103/PhysRevResearch.3.023178

\section{INTRODUCTION}

The two pillars of modern physics, quantum mechanics and general relativity, are expected to be eventually combined into the elusive theory of quantum gravity (QG) [1-3]. However, while separately the two theories are well tested, the former in the regime of large masses and distances and the latter in the microscopic regime, no experiment has been able to probe them simultaneously [4]. To facilitate this formidable task one promising approach is the development of low-energy (infrared) QG phenomenology which could eventually, upon experimental realization, lead to critical experimental hints. Of course, gravity has been extensively probed in the domain of quantum field theory in "classical" curved spacetime $[5,6]$. There the source of the gravitational field is classical and the probe is quantum mechanical. The most notable result is given by the Colella-Overhauser-Werner experiment [7], which has over the years lead to several important matter-wave interferometers [8-10] as well as to more recent developments in photon interferometry [11-13].

To reveal quantum features of the gravitational field one promising approach is to prepare a nonclassical state of a

\footnotetext{
*s.bose@ucl.ac.uk

Published by the American Physical Society under the terms of the Creative Commons Attribution 4.0 International license. Further distribution of this work must maintain attribution to the author(s) and the published article's title, journal citation, and DOI.
}

massive system, resulting in a quantum source of the gravitational field. Specific proposals have been devised to witness the entanglement between two masses mediated through a gravitational field $[14,15]$. As a classical mediator cannot entangle two masses [16], gravity, the mediator of the above entanglement, must be quantum $[14,17,18]$. This seems to be currently the only conclusive way to witness the fundamentally quantum nature of gravity in the laboratory. Each mass is placed in a superposition of two positions, which can be rephrased in a suggestive way by employing a general relativistic viewpoint: it is a superposition of spacetime geometries [19].

An important question is the level of ambient noise under which interference or entanglement can be detected. This has been estimated under generic amounts of decoherence [20,21] and mitigating pressures and temperatures have been estimated for gas collisions and blackbody sources [14,22]. However, any large-mass matter-wave experiment, which requires large momentum transfer to achieve sufficient wave function splitting, is acutely susceptible to acceleration noise [23,24]. Recently, it has been claimed that this type of noise acutely affects the witnessing of entanglement [25] in the quantum nature of gravity experiment [14].

Here we propose how the two universal dephasing channels_-noninertial jitter (i.e., residual acceleration noise) and gravity-gradient noise (GGN) - that will limit any large momentum transfer matter-wave interferometry experiment can be mitigated. Large momentum transfer will always be required for scaling matter-wave interferometry to large masses, for which there is wide motivation: not only the quantum 
nature of gravity as mentioned above, but also quantum sensing [26-28] and testing the ultimate limits of quantum mechanics [29]. Both effects induce random relative accelerations between the interfering paths as well as with the experimental apparatus (the control fields and beam splitters that create the superposition as well as the measuring devices), resulting in a loss of visibility.

We obtain simple formulas to describe the loss of coherence due to noninertial jitter, induced by gas collisions and photon scattering on the experimental container, and gravity gradients, induced by external masses and the intrinsic finite size of matter-wave systems. In particular, the derived expressions depend only on generic properties of any matter-wave experiment and are independent of the specific mechanism or protocols to prepare and recombine the superpositions. In addition, we estimate the effects for the interferometric setup from [14] and show that this experiment can be made insensitive to the above two universal noise effects by controlling the environment (see Figs. 3 and 5). The noninertial jitter of the experimental apparatus (in particular, uncontrollable motion of the magnets) gives rise to an acceleration noise which has to be kept below $\sim \mathrm{fm} \mathrm{s}^{-2} / \sqrt{\mathrm{Hz}}$ [25]; we find such a value can be achieved by placing the experiment in a vacuum chamber with pressure $\sim 10^{-6} \mathrm{~Pa}$ or lower (one can have further technical noises, e.g., vibrations, rotational noise, charge noise, which have to be controlled to the same degree, but similarly do not pose a fundamental limitation). Gravity-gradient noise can be on the other hand mitigated by limiting access to the immediate vicinity of the experiment to massive moving bodies (e.g., to $\sim 5 \mathrm{~m}$ for humans, $\sim 10 \mathrm{~m}$ for cars, and $\sim 60 \mathrm{~m}$ for planes).

This work is organized in the following way. We discuss how to derive the Lagrangian appropriate for interferometric protocols starting from Fermi-normal coordinates (Sec. II). We then discuss phase accumulation in single-particle interferometry experiments [26], in particular, focusing on noninertial jitter and the resulting loss of visibility. We then discuss the gravity-gradient noise (GGN) due to finite-size effect of the capsule and outline its mitigation (Sec. III). We finally apply the results to the experimental setup to detect the quantum nature of gravity [14] where we consider an improved scheme to reduce the Casimir-Polder interaction [22]. Here we give quantitative estimates for the entanglement witness under feasible mitigations of the above noise sources using a recently proposed improved entanglement witness [21] (Sec. IV).

\section{REFERENCE FRAMES AND MATTER WAVES}

A convenient coordinate system to describe matter-wave experiments is the one where the experimental equipment remains stationary (see Fig. 1). Assuming that the experimental apparatus is attached to a container-forming an experimental box-one can consider the motion of its center of mass and construct the associated timelike curve in spacetime. Here we are assuming that the mass of the system, $m$, is much smaller then the total mass of the experimental box, $M$, such that the effect of the former on the latter can be neglected. To describe such nearly local interferometric experiments we first construct Fermi-normal coordinates (FNCs). In particular, the

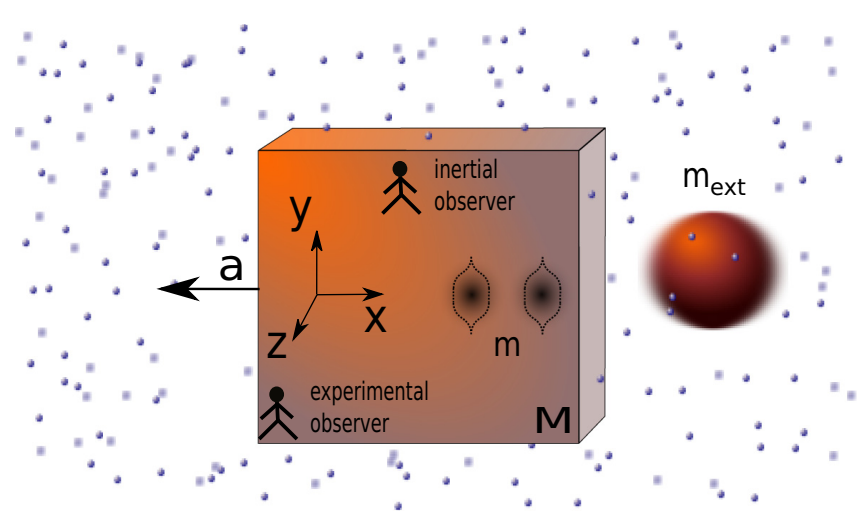

FIG. 1. Conceptual scheme of the experiment as seen by a distant observer. Here we focus on the horizontal $x$ motion where the objects of mass $m$ are placed in a spatial superposition. Both the system (here depicted as two adjacent interferometers) and the experimental apparatus (here illustrated as a box of mass $M$ ) follow approximately geodetic motion. The deviation from ideal geodetic motion is due to gas collisions and photon scattering (here we have illustrated only dust particles outside the experimental container). To describe the experiment we consider an ideal free-falling observer and an observer attached to the experimental container. For the ideal free-falling observer also the experimental box becomes a dynamical degree of freedom (to account for its motion about the geodesic), while the observer attached to the experimental container will describe it using an accelerated reference frame with a time-dependent acceleration $a$. In addition, any external mass $m_{\text {ext }}$ will generate a small gravity gradient over the finite extension of the experiment. On the other hand, the uniform potential generated by the same mass $m_{\mathrm{ext}}$ vanishes due to the equivalence principle: for the experimental observer both the system and the apparatus fall at the same rate toward any external mass.

FNC metric is given by $[30,31]$

$$
\begin{gathered}
d s^{2}=g_{t t} c^{2} d t^{2}+2 g_{t b} c d t d x^{b}+g_{b c} d x^{b} d x^{c}, \\
g_{t t}=-\left[\left(1+a_{b} x^{b}\right)^{2}+R_{0 c 0 d} x^{c} x^{d}\right], \\
g_{t b}=-\frac{2}{3} R_{0 c b d} x^{c} x^{d}, \\
g_{b c}=\delta_{b c}-\frac{1}{3} R_{b c d e} x^{d} x^{e},
\end{gathered}
$$

where we have omitted cubic displacements $O\left(x^{3}\right)$ from the reference timelike curve, and $\boldsymbol{a}=\left(a_{1}, a_{2}, a_{3}\right)$ is the acceleration of the observer. The curvature effects are encoded in the Riemann tensor $R$ which can be estimated from the background stress-energy tensor. Here we are also implicitly assuming that the reference frame is not rotating as we have restricted the discussion only to linear accelerations. The FNC construction is typically applied to investigate classical Earthbound experiments as well experiments in free fall [32].

For nonrelativistic matter-wave experiments we can make further approximations. In particular, for slowly moving matter only the $g_{t t}$ term will be important, i.e., when expanding the dynamics to order $O\left(c^{-1}\right)$. We thus approximate the metric in Eq. (1) to

$$
d s^{2}=-\left[\left(1+a_{b} x^{b}\right)^{2}+R_{0 c 0 d} x^{c} x^{d}\right]^{2} c^{2} d t^{2}+\delta_{b c} d x^{b} d x^{c} .
$$


In many cases the curvature effects are negligible; i.e., we can further neglect the Riemann tensor term $\sim R_{0 c 0 d}$ in Eq. (5), resulting in the Rindler metric. We however keep the term $R_{0 c 0 d} x^{c} x^{d}$, which corresponds to Newtonian spacetime curvature, i.e., the gravity-gradient term, which can result in relative accelerations between the mass and the measuring apparatus if they are finitely spatially separated in the laboratory. We keep this in order to examine the influence of GGN, but as we will show, it can be mitigated for all reasonable unknown masses that cannot be tracked during the experiment.

In any case, we can readily write down the Lagrangian of a point particle:

$$
L=-m c^{2} \sqrt{-g_{\mu \nu} \frac{d x^{\mu}}{c d t} \frac{d x^{\nu}}{c d t}},
$$

where $x^{\mu}=(c t, \boldsymbol{x})$ are the FNC coordinates. Since we are primarily interested in the motion along the horizontal direction, i.e., the axis of the spatial superposition, we will in the following omit the coordinates $x_{2}, x_{3}$ and relabel $x_{1}\left(a_{1}\right)$ as $x$ (a). Using the metric in Eq. (5) and the Lagrangian in Eq. (6) we then readily obtain

$$
L=\frac{1}{2} m v^{2}-\max -\frac{1}{2} m \omega_{\mathrm{gg}}^{2} x^{2},
$$

where we have omitted the constant term $m c^{2}$, and we have introduced $\omega_{\mathrm{gg}}^{2}=R_{0101} c^{2}$. The harmonic frequency $\omega_{\mathrm{gg}}$ is associated with the Newtonian gravity-gradient potential due to finite size of the experiment: for an attractive one it is real-valued, but for a repulsive one it becomes imaginary. Physically this corresponds to tidal forces that are compressing or stretching a body, respectively.

We now concentrate on the setting of Fig. 1. The whole experiment is enclosed in a free-fall laboratory (which we also interchangeably call the capsule or box). The Lagrangian we have obtained in Eq. (7) describes the motion of the system from the viewpoint of the noninertial laboratory observer (i.e., comoving with the experimental box). It is important to note that the acceleration $a$ can only result from electromagnetic interactions but not through the gravitational one, e.g., dust particles or photons hitting the experimental box. Importantly, a laboratory interacting only gravitationally with external masses would still result in free fall with vanishing acceleration, i.e., $a=0$. Indeed, from the viewpoint of a distant inertial observer both the experimental box as well as the system would be accelerating toward the external mass with the same acceleration, $G m_{\text {ext }} / R^{2}$, where $m_{\text {ext }}$ is the mass of the external object, and $R$ is the distance between the external object and the center of the experimental box. On the other hand, gravity-gradient potentials here parametrized by $\omega_{\mathrm{gg}}$, cannot be eliminated by simple change of coordinates, as quantum-mechanical systems are always of finite extension due to their wave nature.

In summary, one can repeat the FNC construction for different observers, following different timelike curves. In this section we have already discussed three different observers, each of which has a different coordinate system: an ideal free-falling observer following a geodesic, the approximately free-falling observer following the timelike curve of the experimental box, and the distant inertial observer fixed with respect to the stars. While the above construction was based on the general relativistic formalism, the same nonrelativistic results can be obtained directly using extended Galilean transformations. Importantly, noninertial effects can be seen as relative motion between the experimental box and the system, the former following a nongeodesic timelike curve while the latter on a geodesic (when in perfect isolation). On top of this, each interaction of a gas particle or a photon with the system will induce nongeodesic motion of the latter: this gives rise to the decoherence already considered in $[14,22]$. On the other hand, gas and photon collisions with the experimental box provide a second mechanism for the loss of visibility: we will refer to it as noninertial jitter (sometimes labeled as residual acceleration noise). However, there is an important difference between the two: unlike decoherence, the loss of visibility stemming from noninertial jitter can, at least in principle, be completely canceled by a control experiment. Indeed noninertial jitter, as well as any other classical deterministic noise, can be measured using a second system, and addressed either by actively recalibrating the experimental apparatus in real time or passively in postanalysis. As we will see in the next section noninertial jitter is a technical challenge, but does not present a fundamental limitation for interferometry with large masses.

\section{NONINERTIAL JITTER AND GRAVITY- GRADIENT NOISE}

In this section we consider a single interferometer for a mass $m$ with two internal states $\mathrm{s}_{j}(j=L, R)$; we create and control the superposition size by using state-dependent forces [14]. In particular, we first create a spatial superposition, maintain it in a fixed size $\Delta x$ for a fixed interval of time, and then recombine it, as shown in Fig. 2; at the end we measure the resulting accumulated phase difference. We describe the two paths of the superposition using the semiclassical approach [33]. We consider the Lagrangian obtained in Eq. (7) and add the interaction for controlling the superposition size. Specifically, for the two paths we have the following Lagrangian:

$$
L_{j}=\frac{1}{2} m v_{j}^{2}-m a(t) x_{j}-m \lambda_{j}(t) x_{j}-\frac{1}{2} m \omega_{\mathrm{gg}}^{2}(t) x_{j}^{2},
$$

where $j=L, R$ denotes the left or right path, $x_{j}$ the particle position, $\lambda_{j}(t)=\frac{f_{\mathrm{m}}}{m} \mathrm{~S}_{j}$ is a state-dependent acceleration generated from a force of amplitude $f_{\mathrm{m}}$ (the internal-state labels can acquire values $s_{j}= \pm 1$ during the creation of the superposition and its recombination, while during the period the superposition is held constant, it is set to $\mathbf{s}_{j}=0$; see Fig. 2), and $a(t)$ is the time-dependent acceleration as described by the noninertial observer attached to the experimental box (here we are using the term noninertial as the box is subject to noninertial jitter). In Ref. [14], a specific realization of the state-dependent force was suggested, where $\mathbf{s}_{j}$ corresponded to NV center spin states in a diamond nanocrystal, and the state-dependent force was generated by a magnetic field gradient $\frac{\partial B}{\partial x}$ through

$$
f_{\mathrm{m}}=g_{N V} \mu_{B} \frac{\partial B}{\partial x},
$$

where $g_{\mathrm{NV}}$ is the electronic $g$ factor, $\mu_{B}$ is the Bohr magneton, and $B$ is the component of the magnetic field along $x$. However, here we are going to refrain from the details of 


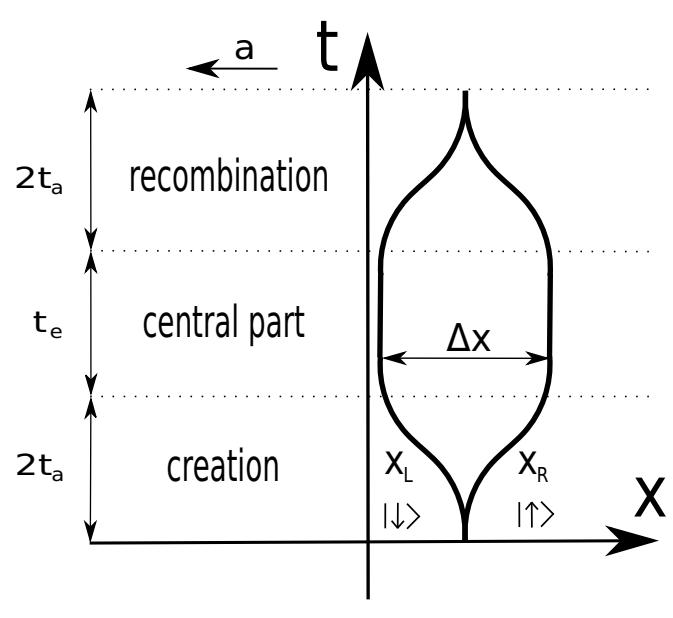

FIG. 2. Paths for a single-particle interferometry experiment as described from the viewpoint of an observer stationary with the experimental box; we have indicated by $a$ the residual acceleration which arises due to the collisions of the experimental box with gas particles (see Fig. 1). The paths are predominantly determined by the magnetic field gradient forces (and hence still symmetric), while the phases can have also unknown random contributions from noninertial and gravity-gradient terms. Such phases can induce a dephasing channel when there is momentum transfer between the system and experimental apparatus (i.e., when there is relative motion between the system and experimental apparatus and the two are coupled). The interferometric loop has three parts: (i) creation of superposition, (i) central part when the system is completely decoupled from the experimental apparatus, and (iii) recombination of the superposition. Interference and dephasing can be discussed only when the full interferometric loop is taken into account (see text).

the properties of the crystal and the source of the magnetic field gradient [34]. Instead, we are going to focus on those relative acceleration noise sources which would be present in any realization of matter-wave interferometry through generic internal-state-dependent forces as modeled in Eq. (8). However, for simplicity of presentation we are going to refer to the internal states as spins.

The trajectories for the two states associated with the different initial position and spin are determined by the simple equation

$$
\ddot{x}_{j}(t)=\lambda_{j}(t)
$$

Here we have omitted the contribution from gravity gradients of unknown external masses, i.e., $\omega_{\mathrm{gg}}^{2}=0$, as we are primarily interested in the trajectories (while the effect of gravity-gradient terms from known sources on the trajectory can be readily taken into account in the analysis and are thus also omitted here). Similarly, unknown sources of $a(t)$ in a controlled environment will be small and can be neglected, while known sources, such as due to the motion of the experimental apparatus, can be fully taken into the analysis. However, the same argument we have applied for trajectories does not apply to the accumulated phase difference where already tiny noninertial and gravity-gradient contributions could rotate it by a substantial fraction of $2 \pi$. This will be discussed in detail below. The trajectories are thus determined by

$$
x_{j}=\int_{0}^{t}\left[\int_{0}^{u^{\prime}} \lambda_{j}(u) d u\right] d u^{\prime},
$$

where we have assumed $x_{j}(0)=0$ and $\dot{x}_{j}(0)=0$.

For a single particle we can generate two distinct paths, i.e., $j=L$ and $j=R$ (left and right paths, respectively), by considering opposite spins, i.e., $\mathrm{s}_{L}=-\mathrm{s}_{R}$, such that the magnetic forces are opposite: $\lambda_{L}=-\lambda_{R}$. The condition to close the loop at time $t=t_{f}$ is given by requiring

$$
x_{L}\left(t_{f}\right)=x_{R}\left(t_{f}\right) .
$$

As the state-dependent force depends linearly on the spin of the particle, i.e., $\propto \mathbf{s}_{j}$, this give a condition on the time dependence of the spin values,

$$
\int_{0}^{t}\left[\int_{0}^{u^{\prime}} \mathrm{s}_{j}(u) d u\right] d u^{\prime}=0,
$$

i.e., the condition to close the interferometric loop. For example, Eq. (13) can be fulfilled by controlling the spins as follows:

$$
\mathrm{s}_{L}(t)= \begin{cases}-1, & 0<t<t_{a} \\ +1, & t_{a}<t<2 t_{a} \\ 0, & 2 t_{a}<t<2 t_{a}+t_{e} \\ +1, & 2 t_{a}+t_{e}<t<3 t_{a}+t_{e} \\ -1, & 3 t_{a}+t_{e}<t<4 t_{a}+t_{e}\end{cases}
$$

with the opposite values for $\mathbf{s}_{R}(t)$. The total experimental time is given by $t_{f}=4 t_{a}+t_{e}$, where we will refer to $t_{a}\left(t_{e}\right)$ as the acceleration (free-fall) time interval. Even if this condition is not exactly met experimentally, as long as the final states are approximately equal, i.e., with nearly overlapping wave packets, one will not have substantial loss of visibility: if the spread of the wave packets is $\sigma$, one requires $\left|x_{L}\left(t_{f}\right)-x_{R}\left(t_{f}\right)\right| \ll \sigma$. Note that a random acceleration $a(t)$ does not affect at all the condition $x_{L}\left(t_{f}\right)=x_{R}\left(t_{f}\right)$ : both paths are subject to exactly the same random acceleration $a(t)$ and the loop thus remains perfectly closed. Only the random fluctuating gravity-gradient term can affect the closed-loop condition when sufficient asymmetry is present in the problem. Indeed, this is SternGerlach interferometry, which has recently been implemented with atoms [35-37] and suggested for large masses [38,39]. We should note, however, this criterion can be difficult to meet, and it is eased by cooling the masses to the ground state initially in a trap, which has already been achieved [40].

In the next two sections we will estimate two dephasing channels using the method which we now sketch. The accumulated phase difference for the interferometric loop is given by

$$
\Delta \phi=\phi_{R}-\phi_{L},
$$

where the accumulated phase over each path $j=L, R$ is given by

$$
\phi_{j}=\frac{1}{\hbar} \int_{0}^{t_{f}} d t L_{j}(t),
$$

and $t_{f}$ is the time of interferometric experiment. The measured phase can be written as $\Delta \phi=\Phi_{\text {eff }}+\delta \phi$, where $\Phi_{\text {eff }}$ would be the phase in absence of dephasing or decoherence channels, 
and $\delta \phi$ is the fluctuating contribution due to noise sources. We will investigate the decay of coherences, $\Gamma \sim \mathbb{E}\left[\delta \phi^{2}\right]$, arising from noninertial jitter and GGN, $a(t)$ and $\omega_{\mathrm{gg}}^{2}(t)$, respectively, where $\mathbb{E}[\cdot]$ denotes the average over different noise realizations. In particular, the condition to witness interference (as well as entanglement) can be cast in the form

$$
\Phi_{\text {eff }}>\Gamma_{\text {jitter }}+\Gamma_{\mathrm{gg}},
$$

where $\Gamma_{\text {jitter }}$ and $\Gamma_{\text {gg }}$ denote the decay of coherences due to noninertial jitter and GGN, respectively (any other channel for the loss of visibility will appear on the right-hand side as an additional contribution).

\section{A. Noninertial noise and residual acceleration noise}

We want to calculate the accumulated phase difference arising from the noninertial jitter of the experimental box (sometimes labeled as residual acceleration noise in the literature) and estimate its effect on the interferometric visibility. ${ }^{1}$ Exploiting the Lagrangian in Eq. (8) and the trajectories given by

$$
x_{j}=\int_{0}^{t}\left[\int_{0}^{u^{\prime}}\left[\lambda_{j}(u)+a(u)\right] d u\right] d u^{\prime},
$$

we eventually find a simple expression

$$
\Delta \phi=\frac{2 m}{\hbar} \int_{0}^{t_{f}} d t \lambda(t) X(t),
$$

where we have defined

$$
X(t)=\int_{0}^{t}\left[\int_{0}^{u^{\prime}} a(u) d u\right] d u^{\prime} .
$$

We note that from the perspective of the inertial observer $X(t)$ corresponds to the displacement of the experimental box about the geodesic trajectory generated by gas collision (the analogous effect generated by photons follows the same analysis).

We can thus readily model the motion of the center of mass of the experimental box as a classical degree of freedom: $X$ $(P)$ will be a classical position (conjugate momentum) observable of the experimental box. Specifically, we have the following stochastic differential equations [41]:

$$
\begin{gathered}
\dot{X}=\frac{P}{M}, \\
\dot{P}=-\Omega^{2} X-\gamma P+\sqrt{2 \gamma M k_{B} T} P_{\text {in }} .
\end{gathered}
$$

The gas-damping coefficient is given by [42]

$$
\gamma=\frac{p l^{2}}{M}\left(1+\frac{\pi}{8}\right)\left(\frac{32 m_{g}}{\pi k_{b} T}\right)^{1 / 2},
$$

where $p(T)$ is the gas pressure (temperature), $m_{g}$ is the mass of a gas molecule, and $l$ is the linear size of a cubic experimental box. Here we have also included for completeness the harmonic frequency, $\Omega$, which has to be taken into account, for example, when the experimental setup is suspended. In

\footnotetext{
${ }^{1}$ The gravity-gradient terms, which will be discussed in the next subsection, are here set to zero, i.e., $\omega_{\mathrm{gg}}=0$.
}

the following we however set it to zero, i.e., $\Omega=0$, as is the case for a free-falling setup. Even if $\Omega$ is nonzero, as long as $\Omega<\omega_{\min }=2 \pi t_{\exp }^{-1}$, where $t_{\exp }$ is the experimental time, we can safely neglect it. $P_{\text {in }}$ is the classical input noise quantified by

$$
\mathbb{E}\left[P_{\text {in }}(t)\right]=0, \quad \mathbb{E}\left[P_{\text {in }}(t) P_{\text {in }}\left(t^{\prime}\right)\right]=\delta\left(t-t^{\prime}\right),
$$

where $\mathbb{E}[\cdot]$ denotes the average over different noise realizations. To describe the noninertial jitter of the experimental box induced by photons one has to a use a modified Eq. (23) but will produce only a subleading effect in a controlled environment-which we leave for future work [43].

From Eqs. (21) and (22) we can readily find the power spectral density (PSD):

$$
S_{X X}(\omega)=\frac{4 k_{B} T}{M} \frac{\gamma}{\left(\Omega^{2}-\omega^{2}\right)^{2}+\omega^{2} \gamma^{2}} .
$$

We note that the noise decreases as $1 / \omega^{4}$ thus strongly suppressing high-frequency noise. From Eqs. (19) and (25) we can now find the fluctuations of the accumulated phase:

$$
\Gamma_{\mathrm{jitter}} \equiv \mathbb{E}\left[\Delta \phi^{2}\right]=\frac{2 m^{2}}{\pi \hbar^{2}} \int_{-\infty}^{\infty} d \omega F_{\mathrm{jitter}}(\omega) S_{X X}(\omega),
$$

where we have defined

$$
F_{\mathrm{jitter}}(\omega)=\left[\int_{0}^{t_{f}} d t \int_{0}^{t_{f}} d t^{\prime} \lambda_{t} \lambda_{t^{\prime}} e^{i \omega\left(t-t^{\prime}\right)}\right] .
$$

Using Eq. (14) the function $F$ is given by

$$
F_{\mathrm{jitter}}(\omega)=\left(\frac{f_{\mathrm{m}}}{m}\right)^{2} \frac{64 \sin ^{4}\left(\frac{\omega}{2} t_{a}\right) \sin ^{2}\left[\frac{\omega}{2}\left(2 t_{a}+t_{e}\right)\right]}{\omega^{2}},
$$

which we note is symmetric in $\omega$.

It is instructive to further explore the regime of low damping as the experiment is expected to be in a controlled environment. In particular, we consider the case when the damping $\gamma$ is small on the timescale of the experiment, i.e., $\omega>\gamma$. We can thus simplify Eq. (25) as

$$
S_{X X}(\omega) \approx \frac{4 k_{B} T}{M} \frac{\gamma}{\omega^{4}} .
$$

We can now use Eqs. (26)-(29), to obtain a simple formula for the phase fluctuations:

$$
\Gamma_{\mathrm{jitter}}=\frac{16 \gamma k_{B} T f_{\mathrm{m}}^{2}}{\hbar^{2} M}\left[\frac{23}{15} t_{a}^{5}+t_{a}^{4} t_{e}\right] .
$$

Let us briefly discuss how to mitigate the phase fluctuations in Eq. (30). Using Eq. (23) we first note that Eq. (30) has the desired behavior with the pressure, $p$, and temperature, $T$, of the environment, and can thus be controlled using cryogenics and vacuum chambers. We can furthermore strongly mitigate the phase fluctuations by lowering the experimental time $t_{\exp }=4 t_{a}+t_{e}$, for example, by running simultaneously a large number of equal experiments. In addition, we note that increasing the mass $M$ of the experimental container also suppress the phase fluctuation; i.e., the jitter of a heavier experimental box will be smaller with respect to a lighter one. Specifically, from Eq. (23) we find that $\gamma$ scales with the area $l^{2}$, and is inversely proportional to the mass, i.e., $\sim l^{2} / M$, and thus the overall scaling is $\sim l^{2} / M^{2} \sim 1 /\left(\rho^{2} l^{4}\right)$, where $l(\rho)$ 
denotes the linear size (average density) of the experimental box.

On the other hand, it is interesting to note that the phase fluctuations in Eq. (30) are completely independent of the particle mass $m$. The only dependence of the matter-wave state is through the superposition size $\Delta x$, which can be seen from the dependency on the state-dependent force $\sim f_{\mathrm{m}}$ and the acceleration or deceleration time interval $\sim t_{a}$; i.e., larger values will make $\Delta x$ larger. Of course if one wants to generate the same superposition size for a heavier particle as the one achieved by a lighter one, $f_{\mathrm{m}}$ would need to be increased by the ratio of their masses. However, for a fixed $f_{\mathrm{m}}$ the loss of visibility is completely independent of the mass of the matter-wave system $m$ : this shows that nanoscale and microscale interferometry presents, as far as noninertial jitter is concerned, the same level of experimental challenge as atomic interferometry.

It is instructive to recast the analysis in terms of an acceleration noise. Specifically, we consider a simplified model for the center-of-mass motion of the experimental box:

$$
\begin{gathered}
\dot{X}=\frac{P}{M}, \\
\dot{P}=M A_{\text {in }},
\end{gathered}
$$

where the input noise is defined by

$$
\mathbb{E}\left[A_{\text {in }}(t)\right]=0, \quad \mathbb{E}\left[A_{\text {in }}(t) A_{\text {in }}\left(t^{\prime}\right)\right]=S_{A A} \delta\left(t-t^{\prime}\right),
$$

$S_{A A}$ is a constant acceleration noise power spectral density, and $\mathbb{E}[\cdot]$ denotes the average over different noise realizations. The more complete model for the center-of-mass motion of the experimental box discussed above [given in Eqs. (21) and (22)] can be formally reduced to the simplified model [given by Eqs. (31) and (32)] by making assumptions similar to those used above [see steps from Eq. (25) to Eq. (29)]. First, one assumes the mechanical frequency of the experimental box, $\Omega$, is vanishingly small (such as in free fall). Second, one assumes that the damping rate, $\gamma$, is small enough such that the damping term, $-\gamma P$, can be omitted. Third, the temperature of the environment, $T$, is very large such that the term $\sqrt{2 \gamma M k_{B} T} P_{\text {in }}$ converges to the finite-force noise term $M A_{\text {in }}$; formally, one is considering an environment in the infinite-temperature limit, $T \rightarrow \infty$, with vanishing damping, $\gamma \rightarrow 0$, such that the product $\gamma T$ remains finite. Anyhow, the simplified model gives in place of Eq. (25) the following displacement spectra:

$$
S_{X X}(\omega)=\frac{S_{A A}}{\omega^{4}} .
$$

By then comparing Eqs. (29) and (34) one can thus extract the following relation:

$$
S_{A A}(\omega) \sim \frac{4 k_{B} \gamma T}{M} .
$$

Specifically, Eq. (35) can be used to estimate the acceleration noise power spectral density from the physical parameters of the problem (such as the ones discussed in Fig. 3). In addition, we use the relation $\Delta x / 2 \sim \frac{f_{\mathrm{m}}}{m} t_{a}^{2}$ [see Fig. 2 and Eqs. (11)-

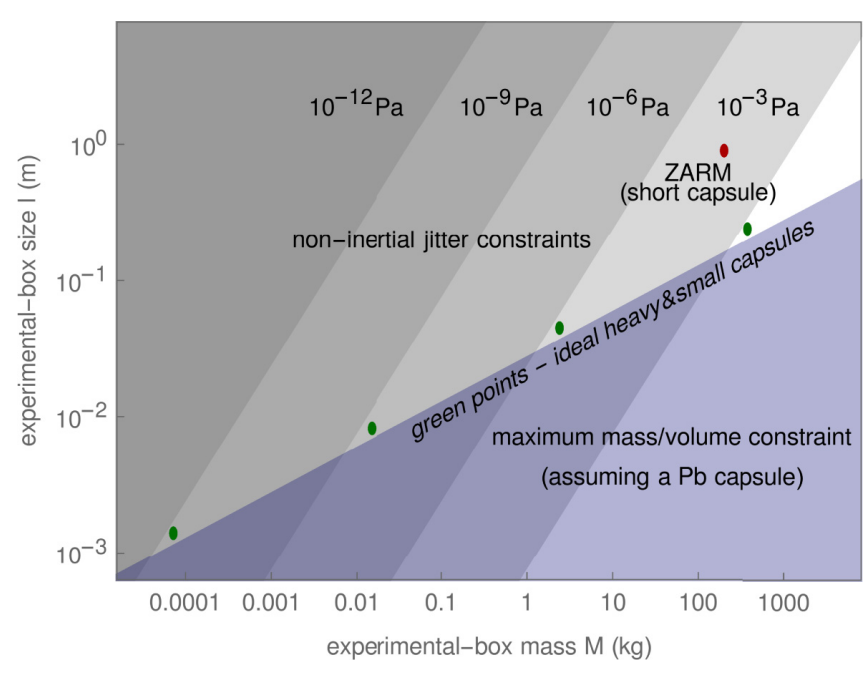

FIG. 3. Plot of the condition to witness entanglement given by $\Phi_{\text {eff }}>\Gamma_{\text {jitter }}(p, T, l, M)$ where $\Phi_{\text {eff }}$ is the effective entanglement phase, and $\Gamma_{\text {jitter }}$ is the damping of coherences due to noninertial jitter; the horizontal (vertical) axis denotes the box mass $M$ (box size $l$, i.e., edge length). Using the experimental values (see Sec. IV) we find the effective entanglement phase $\Phi_{\text {eff }} \sim 0.01$ which gives the constraint $\Gamma_{\mathrm{gg}} \ll 0.01$. We consider the outside of the experimental box to be at room temperature, $T=300 \mathrm{~K}$, and set the pressure, $p$, to the following values, $10^{-3} \mathrm{~Pa}, 10^{-6} \mathrm{~Pa}, 10^{-9} \mathrm{~Pa}$, and $10^{-12} \mathrm{~Pa}$; the corresponding excluded parameter space is depicted in shades of gray. The pressure inside the box is set to $\sim 10^{-16} \mathrm{~Pa}$ and hence its effect on center-of-mass motion of the experimental box can be neglected. In addition, we have colored in light blue the region which would require capsule densities larger than of lead $(\mathrm{Pb})$; the allowed parameter regime is thus restricted to the upper part of the plot. We find that noninertial jitter is successfully suppressed as long as the pressure, $p$, is low enough for a given box mass and size. Ideally we would like to have the lightest capsule mass, $M$, which would allow for simple experimental manipulation; we have indicated ideal small and heavy capsules by green points. The limiting cases are given by a $\sim 1.5 \mathrm{~mm}(\sim 25 \mathrm{~cm})$ size capsule which would require $10^{-12} \mathrm{~Pa}$ $\left(10^{-3} \mathrm{~Pa}\right)$ depicted by the green dot in lower left (upper right) corner. We have also indicated in the plot a reference point corresponding to the ZARM short capsule, i.e., $l \sim 0.9 \mathrm{~m}$ and $M \sim 200 \mathrm{~kg}$ [44], well inside the allowed parameter space at $p \sim 10^{-6} \mathrm{~Pa}$. Lowering the outside pressure and temperature would even further relax the constraints on the mass and size of the experimental box.

(14)] to finally rewrite Eq. (30) as

$$
\Gamma_{\mathrm{jitter}} \sim \frac{S_{A A} m^{2} \Delta x^{2}\left(t_{\mathrm{e}}+\frac{23}{15} t_{\mathrm{a}}\right)}{\hbar^{2}} .
$$

Noninertial jitter (i.e., residual acceleration noise) has been recently discussed in [25]; there they have derived an expression for the loss of coherences formally matching Eq. (36) in case $t_{\mathrm{e}} \gg \frac{23}{15} t_{\mathrm{a}}$ (see Eq. (7) in [25]). The derivation of $\Gamma_{\mathrm{jitter}}$ in [25] was based solely on the central part of the interferometric loop without considering the preparation and recombination of the superposition (see Fig. 2) which resulted in the need to apply a somewhat arbitrary frequency filter $\tau^{2} /\left(1+\omega^{2} \tau^{2}\right)$, with a free parameter, $1 / \tau$, which has been later set to match the inverse of the evolution time in the central part, i.e., $\tau \sim t_{\mathrm{e}}$; this procedure has yielded an additional factor $1 / 4$ with respect to 
the full calculation we have used above (which considered the full interferometric loop).

One can take two approaches regarding the value of $S_{A A}$. The approach taken in [25] is to extract its value from existing or proposed experiments $[45,46]$. However, to devise tailor-made nanocrystal matter-wave experiments a preferred choice is to develop an underlying theoretical model which captures the physics of the residual acceleration noise; this is the approach taken in this work. We have shown explicitly that the noninertial jitter cannot be induced by unknown external masses ${ }^{2}$-in full accordance with the equivalence principle - but rather is of electromagnetic origin (dust particles or photons hitting the experimental apparatus) and can thus be successfully reduced in a controlled environment. Importantly, using Eqs. (35) and (36) one can design matter-wave interferometry experiments with nano- and microsize particles; specifically, one can find the requirements on the experimental box (size and mass) and on the environment (pressure and temperature) to successfully perform the experiment. $^{3}$

\section{B. Gravity-gradient noise due to finite-size effects}

Gravity-gradient noise (GGN), as described by Eq. (5), will arise from stochastic variations in the curvature which remain as an external gravitational signal even in a nearly local experiment due to its finite size. In place of the Lagrangian in Eq. (8) we now consider

$$
L_{j}=\frac{1}{2} m v_{j}^{2}-m \lambda_{j}(t) x_{j}-\frac{1}{2} m \omega_{\mathrm{gg}}^{2}(t) x_{j}^{2},
$$

where we assume $\omega_{\mathrm{gg}}^{2}(t)$ is a multiplicative noise. Here we are considering only the GGN from movements of untracked external masses, while any contributions from known masses can be measured and taken into account in the analysis without any loss of visibility. Here we omit the linear acceleration term $\sim a(t)$, which models the noninertial jitter, and has been already discussed in Sec. III A.

GGN on free test masses has primarily been estimated in the gravitational wave detection literature [47-50], from which, instead of $\omega_{\mathrm{gg}}^{2}(t)$, which is the key quantity relevant for us, it is the random accelerations $a_{\text {rand }}$ of test masses, which is readily available. The calculations of $a_{\text {rand }}$ are based on the cumulative Newtonian effect of environmental mass movement noises on a free test mass, but quite independent of the specifics of gravitational wave detectors, so that it is readily usable in our case. While this acceleration noise $a_{\text {rand }}$ itself will be completely eliminated in our proposed freefall laboratory, it can be used to estimate the noise in $\omega_{\mathrm{gg}}^{2}$,

\footnotetext{
${ }^{2}$ There is gravitational noise due to external masses, but this is a higher-order effect which arises from gravity gradients and the finite size of the experiments (see Sec. III B for more details).

${ }^{3}$ We have shown that noninertial jitter (i.e., residual acceleration noise) is not a fundamental limitation of the proposed quantumgravity-entangling-of-masses scheme [14]—or for this matter for any nano- and microscaled interferometric scheme-but rather can be mitigated by considering a heavy experimental box (sometimes referred to as a capsule) in a low-pressure environment (see Fig. 3).
}

following Ref. [51], to give

$$
S_{\omega_{\mathrm{gg}}^{2} \omega_{\mathrm{gg}}^{2}}(\omega) \equiv \frac{1}{\bar{r}^{2}} S_{a_{\mathrm{rand}} a_{\mathrm{rand}}}(\omega)=\frac{\bar{a}^{2}}{\bar{r}^{2}} \frac{1}{\left(\frac{\omega}{C}\right)^{\alpha}},
$$

where we have introduced the strength of the local acceleration fluctuations, $\bar{a}$, a length-scale parameter $\bar{r}$ characterizing the distance to the GGN sources, and a decay integer $\alpha>1$ which depends on the type of source. $C=2 \pi \times 1 \mathrm{~Hz}$ is a constant that fixes the correct dimensions. In a more refined analysis one would need to consider all external masses, and their associated stochastic motions, which would determine the value of $\frac{\bar{a}^{2}}{\bar{r}^{2}}$ as well as of $\alpha$ in Eq. (38); for a fixed value $\bar{a}$ one can interpret $\bar{r}$ as a characteristic length scale of all the GGN sources combined [47].

It is instructive to obtain an upper bound on the noise spectrum $S_{\omega_{\mathrm{gg}}^{2} \omega_{\mathrm{gg}}^{2}}(\omega)$ by considering the smallest possible distance from the experiment $r_{\text {min }}$ which could contain the bulk of the GGN sources. The environment around the experiment, i.e., located at $r<r_{\min }$, can be well controlled by the experimentalists (for example, it could correspond to the inside of the building in a drop-tower experiment), and as such will not contribute to GGN. On the other hand, any external mass at distances larger than $r_{\min }$ will give a smaller contribution to the GGN than it would if its motion reached $r_{\text {min }}$. In other words, here we will assume that all of the GGN sources reach the outer perimeter of the controlled laboratory environment; i.e., we will set $\bar{r}=r_{\min }$ in Eq. (38), which will give an upper bound on the noise. In practice, the noise will be significantly smaller, with $\bar{r} \gg r_{\min }$.

We can find the phase fluctuations induced by GGN following a similar analysis to that in Sec. (III A). In the first instance we can assume that the trajectory for $x_{j}(t)$ is given by Eq. (11), solely determined by the magnetic gradients. From Eqs. (15), (16), and (37) we then readily find the accumulated phase difference:

$$
\Delta \phi=\frac{m}{2 \hbar} \int_{0}^{t_{f}} d t \omega_{\mathrm{gg}}^{2}(t)\left[x_{R}(t)^{2}-x_{L}(t)^{2}\right] .
$$

Interestingly, for symmetric paths with respect to the origin of the coordinate system, i.e., $x_{L}(t)=-x_{R}(t)$, we do not have any accumulated phase difference, i.e., $\Delta \phi=0$, even for a randomly fluctuating $\omega_{\mathrm{gg}}^{2}(t)$; this is a direct consequence of the harmonic form of the gravity gradient potential. On the other hand, if we consider asymmetric paths with respect to the origin of the coordinate system we will have a nonzero value $\Delta \phi$.

For example, when one considers the dynamics in Eq. (10) with the initial condition $\tilde{x}_{j}(0)=\frac{d}{2}$ and $\dot{\tilde{x}}_{j}(0)=0$ one finds the trajectories given by

$$
\tilde{x}_{j}(t)=x_{j}(t)+\frac{d}{2},
$$

where $x_{j}(t)$ is the trajectory given by Eq. (11), i.e., the trajectory with initial condition $x_{j}(0)=0$ and $\dot{x}_{j}(0)=0$. Using $\tilde{x}_{j}(t)$ in place of $x_{j}(t)$ in Eq. (39), as well as the property 
$x_{L}(t)=-x_{R}(t)$, we then find

$$
\Delta \phi=\frac{m d}{\hbar} \int_{0}^{t_{f}} d t \omega_{\mathrm{gg}}^{2}(t) x_{R}(t) .
$$

The gravity-gradient fluctuations can be then obtained from $\mathbb{E}\left[\Delta \phi^{2}\right]$ following analogous steps to those in Sec. III A where we discussed noninertial jitter, and $\mathbb{E}[\cdot]$ denotes the average over different noise realizations. Specifically, we eventually find the following gravity-gradient fluctuations:

$$
\Gamma_{\mathrm{gg}} \equiv \frac{m^{2} d^{2}}{\pi \hbar^{2}} \int_{\omega_{\min }}^{\infty} S_{\omega_{\mathrm{gg}}^{2} \omega_{\mathrm{gg}}^{2}}(\omega) F_{\mathrm{gg}}(\omega),
$$

where

$$
F_{\mathrm{gg}}(\omega)=\int_{0}^{t_{f}} d t \int_{0}^{t_{f}} d t^{\prime} e^{i \omega\left(t-t^{\prime}\right)} x_{R}(t) x_{R}\left(t^{\prime}\right),
$$

we have introduced a low frequency cutoff $\omega_{\min }=2 \pi t_{\exp }^{-1}$, and $t_{\text {exp }}$ is the experimental time.

Using the trajectories in Eq. (10) we can explicitly evaluate Eq. (43):

$$
\begin{aligned}
F_{\mathrm{gg}}(\omega)= & \frac{f_{\mathrm{m}}^{2}}{m^{2}} \frac{e^{-i \omega\left(2 t_{a}+t_{e}\right)}}{\omega^{6}}\left[t_{a}^{2} \omega^{2}+\left(-1+e^{i t_{a} \omega}\right)^{2} e^{i t_{e} \omega}\right] \\
& \times\left[t_{a}^{2} \omega^{2} e^{i \omega\left(2 t_{a}+t_{e}\right)}+\left(-1+e^{i t_{a} \omega}\right)^{2}\right],
\end{aligned}
$$

which we can further approximate as

$$
F_{\mathrm{gg}}(\omega)=\frac{f_{\mathrm{m}}^{2}}{m^{2}}\left[t_{a}^{4}\left(t_{a}+t_{e}\right)^{2} \theta\left(1-t_{a} \omega\right)+\frac{t_{a}^{4}}{\omega^{2}} \theta\left(t_{a} \omega-1\right)\right],
$$

where we have smoothed over the fast-oscillating terms $[\theta$ is the Heaviside step function, i.e., $\theta(x)=0$ for $x<0$ and $\theta(x)=1$ for $x>0$ ].

Using Eqs. (38), (42), and (45), and keeping only the dominant term $\sim t_{\exp }^{\alpha-1}$, we then obtain a simple formula for the gravity-gradient phase fluctuations:

$$
\Gamma_{\mathrm{gg}} \approx \frac{2 \bar{a}^{2} f_{\mathrm{m}}^{2} t_{a}^{4}}{\hbar^{2}}\left(\frac{d}{\bar{r}}\right)^{2}\left[\frac{C^{\alpha}\left(t_{a}+t_{e}\right)^{2} t_{\mathrm{exp}}^{\alpha-1}}{(2 \pi)^{\alpha}(\alpha-1)}\right] .
$$

One first notices that the phase fluctuations in Eq. (46) scale very favorably with the acceleration time, $t_{a}$, as $\sim t_{a}^{4}$ (for the most interesting case $t_{e} \gg t_{a}$ ), much more favorably than with the amplitude of the magnetic force, $f_{\mathrm{m}}$, which scales only as $f_{\mathrm{m}}^{2}$. However the latter parameters also determine the superposition size $\Delta x \propto f_{\mathrm{m}} t_{a}^{2}$, and for a fixed superposition size there is no benefit of reducing $t_{a}$ at the cost of increasing $f_{\mathrm{m}}$.

We also note that $\Gamma_{\mathrm{gg}}$ in Eq. (46) does not depend on the mass of the system, $m$, or any other property of the system. In other words, the phase fluctuations in Eq. (46) are exactly the same for microscale objects as for, say, atoms. Of course the superposition size, $\Delta x$, scales as $\sim f_{\mathrm{m}} / m$ and thus the superposition size will be smaller for a heavier object than would be achieved with a lighter one [compare with phase fluctuations due to noninertial jitter in Eq. (30) which exhibit a similar behavior].

In this section we have considered the stochastic phase fluctuations $\Delta \phi$ in Eq. (41) which then lead to the average effect $\sim \mathbb{E}\left[\Delta \phi^{2}\right]$ in Eq. (46); these arise from the trajectories in Eq. (11), solely determined by magnetic forces. In particular, one finds that $\Delta \phi \propto f_{\mathrm{m}} \omega_{\mathrm{gg}}^{2}(t)$; i.e., the GGN, $\omega_{\mathrm{gg}}^{2}(t)$, is amplified by the coupling to the magnetic force, $f_{\mathrm{m}}$. However, there are also tiny corrections to the trajectories due to noninertial jitter and due to the gravity-gradient forces. In particular, in place of Eq. (10) one has a modified dynamics, i.e., $\ddot{x}_{j}(t)=$ $\lambda_{j}(t)+a(t)+\omega_{\mathrm{gg}}^{2}(t) x_{j}$, where the last two terms on the righthand side are small. Considering the trajectories perturbed by the noises $a(t)$ and $\omega_{\mathrm{gg}}^{2}(t)$ one finds additional contributions to the phase fluctuation $\Delta \phi$. For example, from Eqs. (15) and (16) one will find contributions proportional to $\sim a(t) \omega_{\mathrm{gg}}(t)$. However, the overall phase fluctuation from such terms will be significantly smaller in comparison to the one in Eq. (41), the latter as discussed amplified by the strong magnetic force, while the former a product of two weak effects. We leave the full assessment of such subleading noises for future work.

We finally make a few remarks on the dependency of $\Gamma_{\mathrm{gg}}$ on the parameter $d$. When considering a single interferometer one can trivially achieve $d=0$, and hence $\Gamma_{\mathrm{gg}}=0$, by placing the particle initially at the center of mass $X$ of the experimental box. Indeed, we recall that $d / 2$ is by construction the initial displacement of the particle with respect to $X$. However, in the next section we will consider two particles in a double interferometric scheme where we will no longer have the possibility to eliminate the GGN phase fluctuations simultaneously on both particles (see Fig. 4). In particular, the two particles are placed initially at $\pm d / 2$ and one can no longer avoid the gravity-gradient phase fluctuations in Eq. (46) by displacing the two particle about the center of mass of the experimental box. One could, for example, place one particle at the center of mass of the experimental box, but the other one would be then located, for example, at $d$, which would result in zero GGN phase fluctuations for the former, but nonzero, larger ones, for the latter. In addition, the value of $d$ will be fixed by other experimental requirements and is not vanishingly small. In short, the GGN phase fluctuations in Eq. (46) can be fully eliminated for a path-symmetric singleparticle interferometer, but will have a nonvanishing effect in the double-interferometric scheme with two particles, which we will consider in the next section.

\section{TESTING QUANTUM GRAVITY USING A SPIN ENTANGLEMENT WITNESS}

It was shown recently that by imposing a modification to the quantum-gravity-entangling-of-masses (QGEM) protocol one can employ a magnetic field gradient two orders of magnitudes lower than suggested in the original proposal, while retaining the same acceleration or free-fall time intervals [22]. As such the new experimental setup has a significant effect on noise reduction. In particular, as shown in Secs. III A and III B the noninertial jitter and GGN are both proportional to the square of the magnetic field gradient, hence resulting in a noise reduction by two orders of magnitude.

We will first briefly go over the modified QGEM protocol and discuss the effect of technical noises and decoherence effects (Sec. IV A). We will then estimate the phase fluctuations induced by noninertial jitter and GGN as well as how they affect the detectability of entanglement (Sec. IV B). In the latter sections we will be following the modified protocol as the requirements on the control parameters such as pressure and 


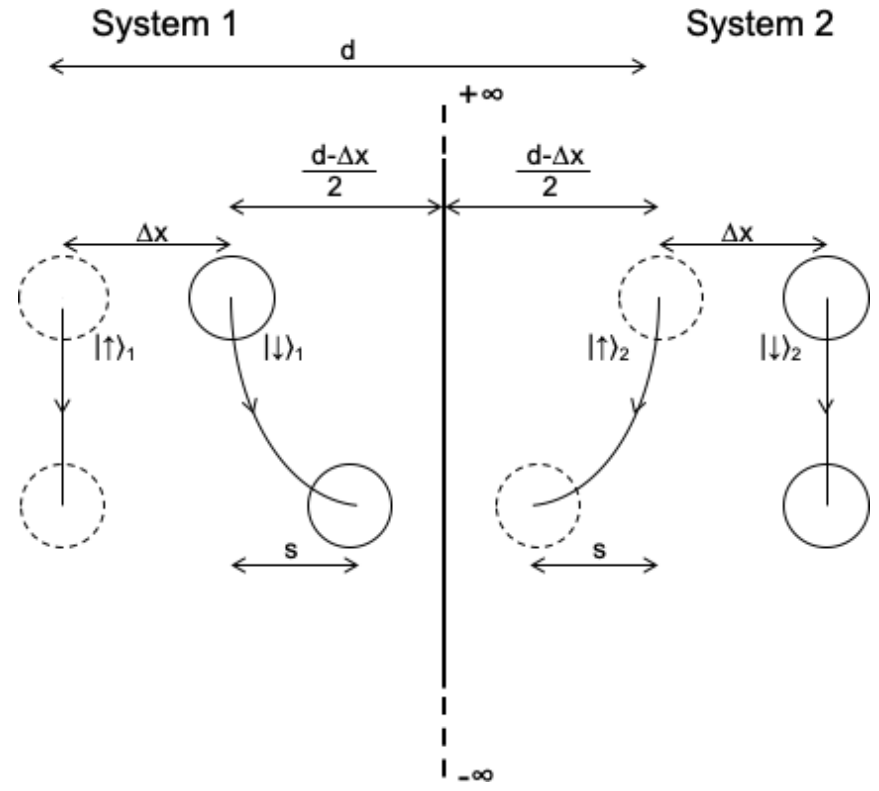

FIG. 4. Modified QGEM protocol. A perfectly conducting plate is placed at the origin which cancels the Casimir-Polder interaction between the two masses, allowing for smaller initial separation, $d$. In particular, one can generate a higher entanglement phase for a given particle mass. However, we have to take into account the deviation of the particle trajectories due to the attractive force with the plate; we denote the displacement of the inner trajectories toward the plate by $s$ (states associated with spins $|\downarrow\rangle_{1}$ and $|\uparrow\rangle_{2}$, where the subscript denotes the particle).

temperature will be less demanding, while the methodology of analysis will resemble the one from the original proposal [14]. We finally briefly comment on the generality of the noise analysis and argue that it can be readily adapted also to other matter-wave experiments (Sec. IV B).

\section{A. Modified QGEM}

The modification of the QGEM protocol recently proposed in [22] is illustrated in Fig. 4. Specifically, a perfectly conducting plate is inserted between the two masses; the plate is fully reflective to electromagnetic waves, which makes it act like a Faraday cage. The two particles can thus no longer interact electromagnetically, even at small relative distances, which significantly relaxes the constraints on their separation; we want the two particles to interact only through the weak gravitational interaction, which is stronger at smaller distances. However, the modification of the boundary conditions for the electromagnetic field produces an attractive Casimir force between the plate and each of the two masses; each mass moves toward the plate during the free fall by a small displacement, $s$.

Let us consider in the first instance only the unitary part of the dynamics during the free-fall time, $t_{e}$, while we neglect all other noises and decoherence channels. One finds that the state at the final time $t_{f}$ would be given by a simple expression

$$
\begin{aligned}
\left|\Psi\left(t_{f}\right)\right\rangle= & \frac{1}{2} e^{i \phi}[|\uparrow\rangle|\uparrow\rangle+|\downarrow\rangle|\downarrow\rangle \\
& \left.+e^{i \Delta \phi_{\uparrow \downarrow}}|\uparrow\rangle|\downarrow\rangle+e^{i \Delta \phi_{\downarrow \uparrow}}|\downarrow\rangle|\uparrow\rangle\right],
\end{aligned}
$$

where $|\cdot\rangle|\cdot\rangle$ is the joint spin state for the two particles, and we have omitted the spatial parts to ease the notation. The accumulated phases are given by

$$
\begin{gathered}
\phi=\frac{G m^{2}}{\hbar} \int_{0}^{t_{e}} \frac{d t}{d-s(t)}, \\
\Delta \phi_{\uparrow \downarrow}=\frac{G m^{2}}{\hbar(d+\Delta x)} t_{e}-\phi, \\
\Delta \phi_{\downarrow \uparrow}=\frac{G m^{2}}{\hbar} \int_{0}^{t_{e}} \frac{d t}{d-\Delta x-2 s(t)}-\phi,
\end{gathered}
$$

where $G$ is the gravitational constant, and $s(t)$ can be determined by the Casimir force induced by the plate [52]. We remark that the Casimir interaction will not give rise to the leakage of "which-path" information into the plate, and, thus, will not be a source of an additional decoherence effect; its effect is fully contained in the displacement $s(t)$. There will of course also be an accumulated phase difference due to the Casimir potential induced by the plate, as its value differs on the inner and outer paths of the individual interferometers. The latter values are however deterministic and can be fully taken into account, but here we choose to omit them for simplicity of presentation. In any case, the parameter which captures the degree of entanglement, namely the effective entanglement phase, is given by $[14,26]$

$$
\Phi_{\mathrm{eff}}=\Delta \phi_{\uparrow \downarrow}+\Delta \phi_{\downarrow \uparrow},
$$

as can be seen by looking at Eq. (47).

We still require that the two interferometric loops remain individually closed, as otherwise no coherence phenomena can be detected. In particular, for a single interferometer we require $\left|x_{L}\left(t_{f}\right)-x_{R}\left(t_{f}\right)\right| \ll \sigma$, where $\sigma$ is spread of the wave packets and $t_{f}=2 t_{a}+2 \tilde{t}_{a}+t_{e}$ is the total time of the interferometer. The acceleration time interval to prepare the superposition is $2 t_{a}$, but now the time to recombine the superposition, $2 \tilde{t}_{a}$, is longer due the the effect of the Casimir plate. Specifically, we have

$$
\tilde{t}_{a}=\sqrt{t_{a}^{2}+\frac{s_{\mathrm{max}}}{a_{\mathrm{m}}}},
$$

where $s_{\max }$ denotes the maximum deviation of the inner trajectories from free fall due to the attractive force toward the plate, $a_{\mathrm{m}}=\frac{f_{\mathrm{m}}}{m}$, and $f_{\mathrm{m}}$ is given in Eq. (9) [see also Eqs. (13) and (14)]. This latter condition poses a limit on the minimum separation between the particles and the plate, $d / 2$, which limits the size of displacement induced by the Casimir plate on the inner paths.

Specifically, we consider two particles with mass $m \sim$ $10^{-15} \mathrm{~kg}$ placed at the distance $d \sim 47 \mu \mathrm{m}$, a magnetic field gradient of $\partial_{x} B=10^{4} \mathrm{~T} \mathrm{~m}^{-1}$, the acceleration time $t_{a} \sim 0.5 \mathrm{~s}$, and the free-fall time $t_{e} \sim 1 \mathrm{~s}$, resulting in a superposition size $\Delta x \sim 23 \mu \mathrm{m}\left(\mu_{B} \sim 9 \times 10^{-24} \mathrm{JT}^{-1}\right.$ and $\left.g_{N V} \sim 2\right)$. By including the full interferometric loop in the analysis (including the creation and recombination parts) one finds $\Phi_{\text {eff }} \sim 0.015$ [22].

The full dynamics however contains also nonunitary contributions as well terms that model technical noises. To describe the final spin state of the two-particle system we construct a joint density matrix with basis elements $|\uparrow\rangle|\uparrow\rangle$, $|\uparrow\rangle|\downarrow\rangle,|\downarrow\rangle|\uparrow\rangle,|\downarrow\rangle|\downarrow\rangle$ which for brevity we will simply 
denote by 1,2,3,4, respectively. A straightforward calculation eventually gives the density matrix $\rho$ at time $t_{f}$, which is defined by the following matrix elements:

$$
\begin{gathered}
\rho_{11}=\rho_{22}=\rho_{33}=\rho_{44}=\frac{1}{4}, \\
\rho_{21}=\rho_{42}^{*}=\frac{1}{4} e^{-\Gamma_{\mathrm{n}} / 2-\Gamma_{d} / 2+i \Delta \phi_{\uparrow \downarrow}}, \\
\rho_{31}=\rho_{43}^{*}=\frac{1}{4} e^{-\Gamma_{\mathrm{n}} / 2-\Gamma_{d} / 2+i \Delta \phi_{\downarrow \uparrow}}, \\
\rho_{41}=\frac{1}{4} e^{-2 \Gamma_{\mathrm{n}}-\Gamma_{d}}, \\
\rho_{32}=\frac{1}{4} e^{-\Gamma_{d} / 2+i\left(\Delta \phi_{\downarrow \uparrow}-\Delta \phi_{\uparrow \downarrow}\right)} .
\end{gathered}
$$

The coherences are now damped; $\Gamma_{\mathrm{n}}\left(\Gamma_{\mathrm{d}}\right)$ is the damping of coherences arising from technical noises (decoherence effects).

\section{Technical noises}

The damping from the technical noises is given by

$$
\Gamma_{\mathrm{n}}=\Gamma_{\mathrm{jitter}}+\Gamma_{\mathrm{gg}},
$$

where $\Gamma_{\mathrm{jitter}}\left(\Gamma_{\mathrm{gg}}\right)$ is given in Eq. (30) [Eq. (46)]. It is important to note that this damping acts only individually on the left and right interferometer; in particular, the degree of entanglement between the two particles, as quantified by the effective entanglement phase $\Phi_{\text {eff }}$ in Eq. (51), remains completely unaltered by $\gamma_{\mathrm{n}}$. Indeed, the gravitationally induced entanglement is due to the correlation of the states $|\uparrow\rangle|\downarrow\rangle$ and $|\downarrow\rangle|\uparrow\rangle$, which gets fully encoded in the matrix elements $\rho_{32}$ and $\rho_{23}$. In other words, technical noises do not change the degree of entanglement, but can only affect the value of a particular entanglement witness. Thus by carefully measuring the noises, for example using a control experiment, one could at least in principle fully counteract their effects thus improving on the interferometric visibility; we leave the investigation of such an active scheme for future research.

Let us briefly describe how to derive the damping arising from technical noises in Eqs. (54)-(56); as discussed in the previous paragraph these can be derived by considering each of the two interferometers individually. In a nutshell, a technical noise will generate a time-dependent, randomly fluctuating phase difference $\Delta \phi$ between the left and right arm of the interferometer [see Eq. (15)]; when considering a large number of runs of the experiment this will reduce the visibility of the coherences. In particular, in place of Eq. (47) we find

$$
\begin{aligned}
\left|\Psi\left(t_{f}\right)\right\rangle= & \frac{1}{2} e^{i \phi^{\prime}}\left[e^{i \Delta \phi}|\uparrow\rangle|\uparrow\rangle+e^{i \Delta \phi_{\uparrow \downarrow}}|\uparrow\rangle|\downarrow\rangle\right. \\
& \left.+e^{i \Delta \phi_{\downarrow \uparrow}}|\downarrow\rangle|\uparrow\rangle+e^{-i \Delta \phi}|\downarrow\rangle|\downarrow\rangle\right],
\end{aligned}
$$

where $\phi^{\prime}$ is a common phase. To find the corresponding statistical operator, averaged over the different runs of the experiment, we calculate $\hat{\rho}=\mathbb{E}\left[\left|\Psi\left(t_{f}\right)\right\rangle\left\langle\Psi\left(t_{f}\right)\right|\right]$, where we assume $\mathbb{E}[\Delta \phi]=0$, i.e., zero-mean fluctuations, and introduce the variance of the fluctuations $\Gamma \sim \mathbb{E}\left[\Delta \phi^{2}\right]$ as discussed in Sec. III.

\section{Decoherence}

The total damping of coherences arising from the decoherence channels is given by [22]

$$
\begin{aligned}
\Gamma_{\mathrm{d}} \equiv & 2 \sum_{j} \Lambda_{j}\left(\frac{46}{15} a_{\mathrm{m}}^{2}\left\{t_{a}^{5}+\tilde{t}_{a}^{5}\right\}+4 a_{\mathrm{m}}^{2} t_{a}^{4} t_{e}\right. \\
& \left.+\int_{0}^{t_{e}}\left[4 a_{\mathrm{m}} t_{a}^{2} s(t)+s(t)^{2}\right] d t\right)+\Lambda_{\text {air }} t_{f} .
\end{aligned}
$$

We have three sources of decoherence, namely, the scattering of air molecules, photon emission and absorption, and photon scattering, quantified by $\gamma_{\text {air }} \equiv \gamma_{\text {air }}\left(p_{\mathrm{e}}, T_{\mathrm{e}}\right), \Lambda_{\mathrm{e}(\mathrm{a})} \equiv$ $\Lambda_{\mathrm{e}(\mathrm{a})}\left(T_{\mathrm{e}(\mathrm{i})}\right)$, and $\Lambda_{\mathrm{sc}} \equiv \Lambda_{\mathrm{sc}}\left(T_{\mathrm{e}}\right)$, respectively, where $T_{\mathrm{e}}\left(p_{\mathrm{e}}\right)$ is the temperature (pressure) inside the experimental box, and $T_{i}$ is the internal temperature of the particle. The explicit expressions can be found in $[53,54]$

$$
\begin{gathered}
\gamma_{\mathrm{air}}=\frac{16 \pi n_{V} R^{2}}{3} \sqrt{\frac{2 \pi k_{B} T_{e x}}{m_{g}}}, \\
\Lambda_{\mathrm{sc}}=8 ! \zeta(9) \frac{8 c R^{6}}{9 \pi}\left(\frac{k_{B} T_{e x}}{\hbar c}\right)^{9} \operatorname{Re}\left(\frac{\epsilon-1}{\epsilon+2}\right)^{2}, \\
\Lambda_{(\mathrm{e}) \mathrm{a}}=\frac{16 \pi^{5} c R^{3}}{189}\left(\frac{k_{B} T_{(i) e x}}{\hbar c}\right)^{6} \operatorname{Im}\left(\frac{\epsilon-1}{\epsilon+2}\right),
\end{gathered}
$$

where $\epsilon$ is the dielectric constant, $n_{V}$ the number density of the gas inside the experimental box, and $r_{s}$ is the sphere radius.

The damping factor $\Gamma_{d}$ in Eq. (60) has been obtained by integrating the effect of the three decoherence sources. Importantly, unlike in the case of noninertial jitter, which is due to the gas on the outside and inside the experimental box, here only the gas environment inside the experimental box decoheres the system. We finally remark that decoherence channels, unlike the technical noises discussed above, affect the density matrix elements $\rho_{32}$ in Eq. (57). Indeed, decoherence presents a fundamental limitation to the degree of entanglement between the two particles, which cannot be removed in a simple way using a control experiment.

\section{B. Detectability of entanglement}

We consider the recently proposed optimized entanglement witness [21]:

$$
\mathcal{W}=\mathbb{I} \otimes \mathbb{I}-\sigma_{x} \otimes \sigma_{x}-\sigma_{y} \otimes \sigma_{z}-\sigma_{x} \otimes \sigma_{z},
$$

where $\sigma_{i}$ are the Pauli matrices. In particular, entanglement is expected to be detected when

$$
\langle\mathcal{W}\rangle=\operatorname{Tr}(\mathcal{W} \rho)<0 .
$$

Using the density matrix elements in Eqs. (53)-(57) we eventually find

$$
\begin{aligned}
\langle\mathcal{W}\rangle= & 1-e^{-\Gamma_{\mathrm{n}} / 2-\Gamma_{\mathrm{d}} / 2}\left[\sin \left(\Delta \phi_{\uparrow \downarrow}\right)+\sin \left(\Delta \phi_{\downarrow \uparrow}\right)\right] \\
& +\frac{e^{-\Gamma_{\mathrm{d}}}}{2}\left[e^{-2 \Gamma_{\mathrm{n}}}+\cos \left(\Delta \phi_{\downarrow \uparrow}-\Delta \phi_{\uparrow \downarrow}\right)\right],
\end{aligned}
$$

where $\Delta \Phi_{\downarrow \uparrow}\left(\Delta \Phi_{\uparrow \downarrow}\right)$ is given in Eq. (49) [Eq. (50)].

If we consider the experimental values in Sec. IV A we find that both $\Delta \Phi_{\downarrow \uparrow}$ and $\Delta \Phi_{\uparrow \downarrow}$ are small, $\sim 0.01$, and hence the damping of coherences, $\Gamma_{\mathrm{n}}$ and $\Gamma_{\mathrm{d}}$, have to be even smaller, i.e., $\Gamma_{\mathrm{n}}, \Gamma_{\mathrm{d}} \ll 0.01$. Hence we can further simplify Eq. (66) 
to obtain a simple expression $\langle\mathcal{W}\rangle=\Gamma_{\mathrm{n}}+\Gamma_{\mathrm{d}}-\Phi_{\text {eff }}$, where $\Phi_{\text {eff }}$ is the effective entanglement phase given in Eq. (51). The condition to witness entanglement is thus $\Phi_{\text {eff }}>\Gamma_{\mathrm{n}}+\Gamma_{\mathrm{d}}$, which in our specific case can be written as

$$
\Phi_{\text {eff }}>\Gamma_{\mathrm{jitter}}+\Gamma_{\mathrm{gg}}+\Gamma_{\mathrm{d}}
$$

i.e., the effective entanglement phase must be larger than the damping of the coherences.

Let us first consider the effect of noninertial jitter while neglecting other channels for the loss of visibility. The condition in Eq. (67) reduces to $\Phi_{\text {eff }}>\Gamma_{\text {jitter }}$. We consider the outside of the experimental box ${ }^{4}$ to be at room temperature $T=300 \mathrm{~K}$ and pressures from $p=10^{-12} \mathrm{~Pa}$ to $p=10^{-3} \mathrm{~Pa}$ (note that while this value of pressure is still lower than the $p=10 \mathrm{~Pa}$ in current drop-tower tubes [55] so that their current microgravity level is not sufficient for us, such pressures, called ultrahigh vacuum, have already been achieved in very large volumes such as in gravitational wave detectors and particle accelerators). In particular, one can observe that for a reasonable mass and size of the box the condition $\Phi_{\text {eff }}>\Gamma_{\text {jitter }}(p, T)$ is satisfied (see Fig. 3); lowering the outside pressure and temperature would even further relax the constraints on the mass and size of the experimental box. One can rewrite the constraint on the noninertial jitter of the experimental apparatus (in particular of the magnets) as a condition on the relative acceleration noise $S_{A A}^{1 / 2}$. Specifically, we recall Eq. (36) which for $t_{\mathrm{a}} \ll t_{\mathrm{e}}$ reduces to $\Gamma_{\mathrm{jitter}} \sim S_{A A} m^{2} \Delta x^{2} t_{\mathrm{e}} / \hbar^{2}$. We further approximate the entanglement phase in Eqs. (49), (50), and (51) as $\Delta \phi_{\text {eff }} \sim G m^{2} t_{e} /(\hbar d)$. Finally, supposing $\Delta x \sim d$ we find that the condition $\Phi_{\text {eff }}>\Gamma_{\text {jitter }}$ reduces to $S_{A A}^{1 / 2} \lesssim \sqrt{G \hbar / d^{3}}$ [25] which for $d \sim 23 \mu \mathrm{m}$ gives $S_{A A}^{1 / 2} \sim 1 \mathrm{fm} \mathrm{s}^{-2} / \sqrt{\mathrm{Hz}}$.

We can also readily estimate the effect of GGN on the detectability of entanglement, while neglecting other channels for the loss of visibility; specifically, the condition in Eq. (67) reduces to $\Phi_{\text {eff }}>\Gamma_{\text {gg }}$. GGN is however highly location dependent and will arise from atmospheric pressure gradients, seismic activity, and anthropogenic sources, among others; such noises cannot be measured directly by gravimeters which record also nongravitational contributions [50], but have to be estimated from atmospheric and geophysical data as well as modeling of anthropogenic activities. Here we will again exploit Eq. (38) which relates the GGN power spectral density (PSD) to the more readily available acceleration noise PSD from the literature [47-49]. For the main GGN sources we will estimate the minimum distance from the experiment, $r_{\min }$, which would still allow the detection of entanglement.

Let us first estimate the GGN contribution arising from the main to nonanthropogenic sources, namely from seismic

\footnotetext{
${ }^{4}$ We consider the internal particle temperature $T_{i}=0.15 \mathrm{~K}$, which we assume to match the temperature inside the experimental box $T_{\mathrm{e}}=1 \mathrm{~K}$, while the pressure inside the box is $p_{\mathrm{e}}=10^{-16} \mathrm{~Pa}$. As these values are substantially lower than the corresponding values outside the experimental box we can safely neglect their effect for noninertial jitter.
}

and atmospheric activity. In particular, we have the following acceleration PSDs [47,48]:

$$
\begin{gathered}
S_{a_{\mathrm{rand}} a_{\mathrm{rand}}}^{\text {atmospheric }}(\omega)=\frac{8 \pi^{3}}{3} G v_{s}^{2} \frac{\rho_{a}^{2}}{p_{a}^{2}} \frac{|\Delta p(\omega)|^{2}}{\omega^{2}}, \\
S_{a_{\mathrm{rand}} a_{\mathrm{rand}}}^{\text {seismic }}(\omega)=\frac{16 \pi^{2}}{3} G \rho_{e}^{2}|\Delta X(\omega)|^{2},
\end{gathered}
$$

for seismic and atmospheric sources, respectively. $v_{s}$ is the speed of sound, $\rho_{a}\left(p_{a}\right)$ is air density (pressure), $\Delta p$ is the pressure fluctuation, $\rho_{e}$ is the ground density near the experiment, and $\Delta X$ is fluctuation of the Earth's surface from the equilibrium position. By integrating over all seismic and atmospheric mass movements as gravitational sources of noise, one can estimate $\bar{a} \sim 10^{-15} \mathrm{~m} \mathrm{~s}^{-2} / \sqrt{\mathrm{Hz}}$ with $\alpha \sim 4$ for $\omega / 2 \pi>10 \mathrm{~Hz}$, and $\bar{a} \sim 10^{-17} \mathrm{~m} \mathrm{~s}^{-2} / \sqrt{\mathrm{Hz}}$ with $\alpha \sim 0$ for $\omega / 2 \pi<10 \mathrm{~Hz}[47,48]$. Using Eq. (46) we find that the condition $\Phi_{\text {eff }}>\Gamma_{\text {gg }}$ is satisfied already if the bulk of the seismic and atmospheric GGN originates at a characteristic distance $r_{\min } \gtrsim 10^{-2} \mathrm{~m}$; this indicates that GGN will likely not be a limiting factor even for drop-tower experiments at the surface of the Earth, as these GGN sources will be far more distant.

Human and anthropogenic movements can also contribute to the GGN-for example, a human walking near the experiment - and one needs to limit access to the experiment within a certain exclusion radius, which we will again indicate with $r_{\min }$. We will consider two classes types of motion: a smooth continuous straight-line motion, and discontinuous acceleration and deceleration jerks. In particular, the acceleration noise PSD generated by an object moving at a constant velocity is given by [47]

$$
S_{a_{\mathrm{rand}} a_{\mathrm{rand}}}^{\mathrm{smooth}}(\omega)=\frac{1}{\omega}\left(\frac{2 G m_{\mathrm{ext}}}{b^{2}}\right)^{2} e^{-2 \frac{b}{v_{\mathrm{ext}}} \omega},
$$

where $v_{\text {ext }}\left(m_{\text {ext }}\right)$ is the speed (mass), and $b$ is the impact factor of the external object. Here we are only interested in finding an upper bound on the GGN fluctuations and will estimate the exclusion zone using the impact factor, i.e., $r_{\min } \sim b$. Although Eqs. (38) and (70) do not lead to the simple formula in Eq. (46), the GGN phase fluctuations in Eq. (42) can be nonetheless readily evaluated numerically. For concreteness, we consider a human of mass $\sim 100 \mathrm{~kg}$ walking at a pace of $\sim 1 \mathrm{~m} \mathrm{~s}^{-1}$, and a car of mass $\sim 1000 \mathrm{~kg}$ driving at $\sim 10 \mathrm{~m} \mathrm{~s}^{-1}$ : we find the exclusion zones $r_{\text {min }} \sim 2 \mathrm{~m}$ and $r_{\text {min }} \sim 10 \mathrm{~m}$, respectively, which can be readily satisfied by restricting access to the experimental building. GGN sources at larger distances do not pose a limiting factor due to the favorable scaling of the GGN fluctuations with the distance from the GGN source, i.e., $\Gamma_{\mathrm{gg}} \sim e^{-2^{\frac{{ }^{\prime} \min }{v}} \omega} / r_{\min }^{6}$. For example, for a plane of mass $\sim 100 \mathrm{t}$ flying at speed $\sim 100 \mathrm{~m} \mathrm{~s}^{-1}$ we find the exclusion radius $r_{\text {min }} \sim 60 \mathrm{~m}$. Humans and cars can contribute to the GGN also in the nonadiabatic regime of sudden acceleration and deceleration; for example, during regular weight transfers between steps. The latter effect can be characterized by the following acceleration noise PSD [49]:

$$
S_{a_{\text {rand }} a_{\text {rand }}}^{\text {jerk }}(\omega)=\frac{16 G^{2} \Delta F_{\text {jerk }}^{2}}{P_{\text {gait }} \Delta t_{\text {jerk }}^{2} r_{\text {min }}^{6} \omega^{8}},
$$




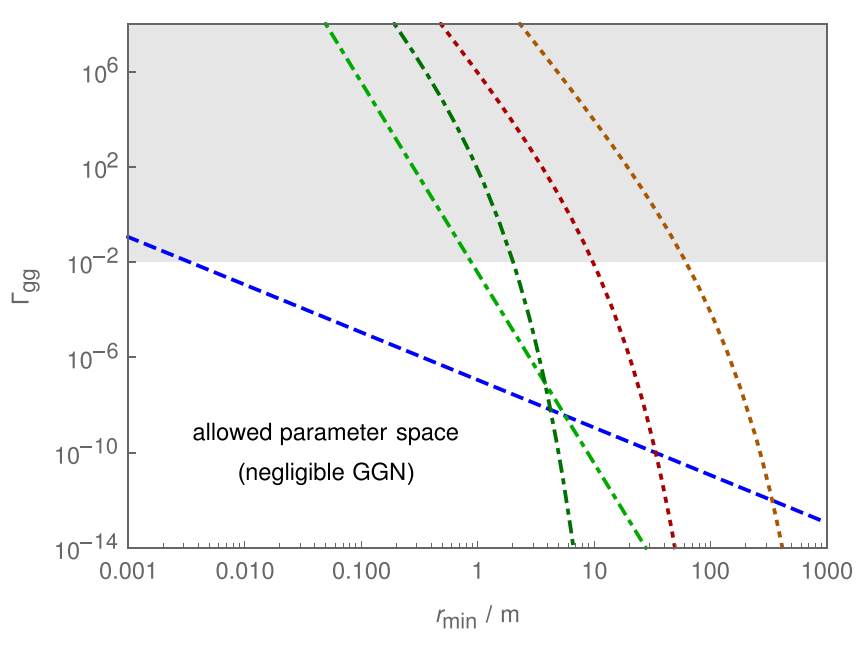

FIG. 5. Plot of GGN phase fluctuations, $\Gamma_{\mathrm{gg}}$, as a function of the minimum allowed distance of the respective GGN source from the experiment, $r_{\min }$, that would still allow the detection of entanglement. Using the experimental values (see Sec. IV) we find the effective entanglement phase $\Phi_{\text {eff }} \sim 0.01$ which gives the constraint $\Gamma_{\text {gg }} \ll$ 0.01 ; the allowed region is the one colored in white in the lower part of the figure. We find that seismic and atmospheric activity does not pose a significant limitation to the experiment (blue dashed line; $r_{\min } \sim 0.01 \mathrm{~m}$ ). Similarly, a human is only restricted from walking in the immediate vicinity of the experiment (light green and dark green dot-dashed lines for a jerking motion and continuous motion, respectively; $r_{\min } \sim 2 \mathrm{~m}$ ). Finally, cars and planes have to be distant by more than $\sim 10 \mathrm{~m}$ and $\sim 60 \mathrm{~m}$, respectively (dark orange and light orange dotted lines, respectively).

where $\Delta F_{\text {jerk }}$ is the change of the horizontal force exerted by the human on the ground in a time interval $\Delta t_{\text {jerk }}$, and $P_{\text {gait }}$ is the gait cycle of two steps. Following Ref. [49] we set $\Delta F_{\text {jerk }} \sim 100 \mathrm{~N}, \Delta t_{\text {jerk }} \sim 20 \mathrm{~ms}$, and consider a step time of $\sim 400 \mathrm{~ms}$ which results in the frequency band $\frac{\omega}{2 \pi} \sim$ [2.5 Hz, $25 \mathrm{~Hz}]$ and the exclusion radius $r_{\min } \sim 1 \mathrm{~m}$. We can also estimate the same effect for a car by assuming a stronger change of force, say $\Delta F_{\text {jerk }} \sim 10^{6} \mathrm{~N} \mathrm{~s}^{-1}$, which however only gives the exclusion radius $r_{\text {min }} \sim 5 \mathrm{~m}$. Indeed, the walking style and pace or the type of car or vehicle will not change drastically the exclusion radius: as can be seen from Eqs. (38) and (71), the GGN phase fluctuations scale as $\Gamma_{\mathrm{gg}} \sim r_{\min }^{-8}$ quickly suppressing the effect of distant sources.

By conducting the experiment in an underground tunnel (still in a free-fall laboratory in the tunnel-the tunnel acting as a drop tower) the GGN can be further reduced. For example, at a depth of $1 \mathrm{~km}$ the typical distance $r_{\min }$ from the source (surface) increases to at least $\sim 1 \mathrm{~km}$, resulting in significantly reduced GGN phase fluctuations $\Gamma_{\mathrm{gg}} \lesssim 10^{-13}$. In summary, we can conclude that in the QGEM experiment the effect of GGN can be fully mitigated (see Fig. 5).

\section{GENERALITY OF ANALYSIS}

The analysis of the fluctuations leading to the dephasing in Eqs. (30) and (46) assumed that a force, $f_{\mathrm{m}}$, is used to create and recombine the superstitions. It is however instructive to rewrite the force in terms of the transferred impulse

$$
\Delta p \equiv f_{\mathrm{m}} t_{a},
$$

where $t_{a}$ is the acceleration time interval. This impulse $\Delta p$ is transferred four times during the creation and recombination parts of the experiments (see Fig. 2). In particular, Eqs. (30) and (46) become

$$
\begin{gathered}
\Gamma_{\mathrm{jitter}} \approx \frac{16 \gamma k_{B} T \Delta p^{2}}{\hbar^{2} M}\left[\frac{23}{15} t_{a}^{3}+t_{a}^{2} t_{e}\right], \\
\Gamma_{\mathrm{gg}} \approx \frac{2 \bar{a}^{2} \Delta p^{2} t_{a}^{2}}{\hbar^{2}}\left(\frac{d}{\bar{r}}\right)^{2}\left[\frac{C^{\alpha}\left(t_{a}+t_{e}\right)^{2} t_{\mathrm{exp}}^{\alpha-1}}{(2 \pi)^{\alpha}(\alpha-1)}\right],
\end{gathered}
$$

respectively. Interestingly, Eqs. (73) and (74) are now independent of the specific coupling between system and apparatus, but depend only on generic experimental matterwave parameters. In particular, Eqs. (73) and (74) depend on the experimental box (mass $M$ and damping $\gamma$ ), the environment outside the box (damping $\gamma$, temperature $T$, the strength of the local acceleration fluctuations $\bar{a}$, a length-scale parameter $\bar{r}$ characterizing the distance to the GGN sources, a decay integer $\alpha>1$ which depends on the type of source, and the constant $C=2 \pi \times 1 \mathrm{~Hz}$ ), the geometry of the paths (separation of the two interferometers $d$ ), the experimental times (acceleration time interval $t_{a}$, evolution time interval $t_{e}$, and total experimental time $t_{\mathrm{exp}}$ ), and finally on the transferred impulse, $\Delta p$. Moreover, as we have already discussed in Sec. III the noise fluctuations $\Gamma_{\mathrm{jitter}}, \Gamma_{\mathrm{gg}}$ do not depend on the mass of the system, $m$. The analysis of the technical noises considered here (noninertial jitter and GGN) is thus quite generic and could be adapted to any matter-wave experiment.

\section{DISCUSSION}

In this paper, we have shown that it is feasible to carry out an experiment on quantum-gravity-induced entanglement of masses (QGEM) terrestrially by going to a freely falling capsule. Of course, carrying out the experiment in space will naturally form such a freely falling laboratory. Under these circumstances, we have investigated the effect of noninertial (i.e., residual acceleration) and gravity-gradient noise on the system that still remains. ${ }^{5}$ These types of noise, if untracked, induce an unknown relative acceleration between the interfering masses and the control and measuring apparatus, which may appear as dephasing. We have thus carefully examined the situations needed to keep the untracked parts below a threshold.

\footnotetext{
${ }^{5}$ There are also other specific systematic noises (i.e., technical noises) depending on the specific mechanism of wave function splitting, some of whose mitigations have already been extensively analyzed [26]. Here we have taken conceptually the simplest route, namely exploiting the equivalence principle to get rid of the bulk of the gravitational noise (all the acceleration noise) so that only relative acceleration noise due to noninertial effects and the finite size of the experiment remains. The other approach of measuring this purely classical noise (see, e.g., [26-28]) and counteracting it by adjusting the detection in real time or in postanalysis will be discussed in a future paper.
} 
We have shown that noninertial jitter (i.e., residual acceleration noise) can only arise from nongravitational effects, e.g., gas particles and photons interacting with the experimental box which make it jiggle about the geodesic motion (see Fig. 1). We have derived the loss of coherence due to noninertial jitter from first principles: the noise originates mainly from the recoil of the experimental box due to collisions with dust particles. Thus noninertial jitter can be successfully mitigated by simply considering a heavy experimental box (sometimes referred as capsule in the text) in a low pressure and temperature environment. The heavier the experimental box, the less it will recoil due to collisions with gas particles, and the lower the pressure, the lower the net recoil of the experimental box; in both cases the residual Colella-Overhauser-Werner phase noise can be strongly suppressed. Specifically, we have shown how different pressure regimes constrain the mass and size of the experimental box to successfully mitigate the corresponding loss of coherence (see Fig. 3). For example, for pressures of $\sim 10^{-6} \mathrm{~Pa}$ outside the freely falling capsule and at room temperature $\sim 300 \mathrm{~K}$, the noninertial component from random molecular kicks on a $\sim 1 \mathrm{~m}$ capsule are low enough to enable a witnessing of the entanglement. Under the above circumstances, for example, we are able to meet the acceleration noise requirement pointed out in [25].

Furthermore, we have shown that the lowest-order gravitational noise arises due to the finite size of the experiment and gravity gradients (such a noise can be seen as the phase counterpart of the tidal forces generated by external masses). We have considered the main sources of gravitational noise: our estimates indicate that gravity-gradient noise from atmospheric and seismic sources is negligible, while anthropogenic contributions can be fully mitigated by limiting access to the immediate vicinity of the experiment (see Fig. 5) - for example, to $\sim 2 \mathrm{~m}$ for humans, to $\sim 10 \mathrm{~m}$ for cars, and to $\sim 60 \mathrm{~m}$ for planes. In summary, noise from gravitational sources can be successfully mitigated by restricting access to the experiment by placing it in a dislocated building or possibly underground.

Finally, we have shown that relative acceleration noise in matter-wave interferometry is intrinsically linked to momentum transfer, $\Delta p$, between the system and experimental apparatus. We have shown how the momentum transfer emerges in the full interferometric loop-in particular, during the preparation and recombination of the superposition when the system and experimental apparatus are coupled (by magnetic fields or otherwise). Thus the dephasing effects discussed in this work will become detrimental in any matterwave interferometry when significant forces and momentum transfers are used [56]; mitigation methods, such as the ones developed in this work, will have to be adopted.

\section{ACKNOWLEDGMENTS}

M.T. and S.B. would like to acknowledge EPSRC Grant No. EP/N031105/1, S.B. EPSRC Grant No. EP/S000267/1, and M.T. funding by the Leverhulme Trust (Grant No. RPG2020-197). A.M.'s research is funded by the Netherlands Organisation for Science and Research (NWO), Grant No. 680-91-119. R.J.M. is funded by a UCL Departmental Studentship. M.S.K. was supported by the EPSRC (Grant No. EP/R044082/1) through the QuantERA ERA-NET Cofund in Quantum Technologies.
[1] D. Oriti, Approaches to Quantum Gravity: Toward a New Understanding of Space, Time, and Matter (Cambridge University Press, Cambridge, UK, 2009).

[2] C. Kiefer, Quantum gravity: General introduction and recent developments, Ann. Phys. 15, 129 (2006).

[3] R. Penrose, On gravity's role in quantum state reduction, Gen. Relativ. Gravit. 28, 581 (1996).

[4] S. Hossenfelder, Experimental Search for Quantum Gravity (Springer, Cham, Switzerland, 2017).

[5] R. Brunetti, C. Dappiaggi, K. Fredenhagen, and J. Yngvason, Advances in Algebraic Quantum Field Theory (Springer, Cham, Switzerland, 2015).

[6] R. M. Wald, Quantum Field Theory in Curved Spacetime and Black Hole Thermodynamics (University of Chicago Press, Chichago, 1994).

[7] R. Colella, A. W. Overhauser, and S. A. Werner, Observation of Gravitationally Induced Quantum Interference, Phys. Rev. Lett. 34, 1472 (1975).

[8] V. V. Nesvizhevsky, H. G. Börner, A. K. Petukhov, H. Abele, S. Baeßler, F. J. Rueß, T. Stöferle, A. Westphal, A. M. Gagarski, G. A. Petrov et al., Quantum states of neutrons in the Earth's gravitational field, Nature (London) 415, 297 (2002).

[9] J. B. Fixler, G. T. Foster, J. M. McGuirk, and M. A. Kasevich, Atom interferometer measurement of the Newtonian constant of gravity, Science 315, 74 (2007).
[10] H. Rauch and S. A. Werner, Neutron Interferometry: Lessons in Experimental Quantum Mechanics, Wave-Particle Duality, and Entanglement (Oxford University Press, Oxford, UK, 2015).

[11] G. Bertocchi, O. Alibart, D. B. Ostrowsky, S. Tanzilli, and P. Baldi, Single-photon Sagnac interferometer, J. Phys. B: At., Mol. Opt. Phys. 39, 1011 (2006).

[12] M. Fink, A. Rodriguez-Aramendia, J. Handsteiner, A. Ziarkash, F. Steinlechner, T. Scheidl, I. Fuentes, J. Pienaar, T. C. Ralph, and R. Ursin, Experimental test of photonic entanglement in accelerated reference frames, Nat. Commun. 8, 15304 (2017).

[13] S. Restuccia, M. Toroš, G. M. Gibson, H. Ulbricht, D. Faccio, and M. J. Padgett, Photon Bunching in a Rotating Reference Frame, Phys. Rev. Lett. 123, 110401 (2019).

[14] S. Bose, A. Mazumdar, G. W. Morley, H. Ulbricht, M. Toroš, M. Paternostro, A. A. Geraci, P. F. Barker, M. S. Kim, and G. Milburn, Spin Entanglement Witness for Quantum Gravity, Phys. Rev. Lett. 119, 240401 (2017).

[15] C. Marletto and V. Vedral, Gravitationally Induced Entanglement between Two Massive Particles Is Sufficient Evidence of Quantum Effects in Gravity, Phys. Rev. Lett. 119, 240402 (2017).

[16] R. Horodecki, P. Horodecki, M. Horodecki, and K. Horodecki, Quantum entanglement, Rev. Mod. Phys. 81, 865 (2009).

[17] A. Belenchia, R. M. Wald, F. Giacomini, E. Castro-Ruiz, Č. Brukner, and M. Aspelmeyer, Quantum superposition of mas- 
sive objects and the quantization of gravity, Phys. Rev. D 98, 126009 (2018).

[18] R. J. Marshman, A. Mazumdar, and S. Bose, Locality and entanglement in table-top testing of the quantum nature of linearized gravity, Phys. Rev. A 101, 052110 (2020).

[19] M. Christodoulou and C. Rovelli, On the possibility of laboratory evidence for quantum superposition of geometries, Phys. Lett. B 792, 64 (2019).

[20] H. C. Nguyen and F. Bernards, Entanglement dynamics of two mesoscopic objects with gravitational interaction, Eur. Phys. J. D 74, 1 (2020).

[21] H. Chevalier, A. J. Paige, and M. S. Kim, Witnessing the nonclassical nature of gravity in the presence of unknown interactions, Phys. Rev. A 102, 022428 (2020).

[22] T. W. van de Kamp, R. J. Marshman, S. Bose, and A. Mazumdar, Quantum gravity witness via entanglement of masses: Casimir screening, Phys. Rev. A 102, 062807 (2020).

[23] S. Bose and G. W. Morley, Matter and spin superposition in vacuum experiment (MASSIVE), arXiv:1810.07045.

[24] J. S. Pedernales, G. W. Morley, and M. B. Plenio, Motional Dynamical Decoupling for Matter-Wave Interferometry, Phys. Rev. Lett. 125, 023602 (2020).

[25] A. Großardt, Acceleration noise constraints on gravity-induced entanglement, Phys. Rev. A 102, 040202 (2020).

[26] R. J. Marshman, A. Mazumdar, G. Morley, P. F. Barker, S. Hoekstra, and S. Bose, Mesoscopic interference for metric and curvature \& gravitational wave detection, New J. Phys. 22, 083012 (2020).

[27] S. Qvarfort, A. Serafini, P. F. Barker, and S. Bose, Gravimetry through non-linear optomechanics, Nat. Commun. 9, 3690 (2018).

[28] F. Armata, L. Latmiral, A. D. K. Plato, and M. S. Kim, Quantum limits to gravity estimation with optomechanics, Phys. Rev. A 96, 043824 (2017).

[29] A. Bassi, K. Lochan, S. Satin, T. P. Singh, and H. Ulbricht, Models of wave-function collapse, underlying theories, and experimental tests, Rev. Mod. Phys. 85, 471 (2013).

[30] C. W. Misner, K. S. Thorne, J. A. Wheeler et al., Gravitation (Macmillan, New York, 1973).

[31] E. Poisson, A. Pound, and I. Vega, The motion of point particles in curved spacetime, Living Rev. Relativity 14, 7 (2011).

[32] C. M. Will, The confrontation between general relativity and experiment, Living Rev. Relativity 9, 3 (2006).

[33] P. Storey and C. Cohen-Tannoudji, The Feynman path integral approach to atomic interferometry. A tutorial, J. Phys. II 4, 1999 (1994).

[34] M. O. Scully, B. G. Englert, and J. Schwinger, Spin coherence and Humpty-Dumpty. III. The effects of observation, Phys. Rev. A 40, 1775 (1989).

[35] S. Machluf, Y. Japha, and R. Folman, Coherent Stern-Gerlach momentum splitting on an atom chip, Nat. Commun. 4, 2424 (2013).

[36] Y. Margalit, Z. Zhou, O. Dobkowski, Y. Japha, D. Rohrlich, S. Moukouri, and R. Folman, Realization of a complete SternGerlach interferometer, arXiv:1801.02708.

[37] O. Amit, Y. Margalit, O. Dobkowski, Z. Zhou, Y. Japha, M. Zimmermann, M. A. Efremov, F. A. Narducci, E. M. Rasel, W. P. Schleich, and R. Folman, $T^{3}$ Stern-Gerlach Matter-Wave Interferometer, Phys. Rev. Lett. 123, 083601 (2019).
[38] M. Scala, M. S. Kim, G. W. Morley, P. F. Barker, and S. Bose, Matter-Wave Interferometry of a Levitated Thermal NanoOscillator Induced and Probed by a Spin, Phys. Rev. Lett. 111, 180403 (2013).

[39] C. Wan, M. Scala, G. W. Morley, A. T. M. A. Rahman, H. Ulbricht, J. Bateman, P. F. Barker, S. Bose, and M. S. Kim, Free Nano-Object Ramsey Interferometry for Large Quantum Superpositions, Phys. Rev. Lett. 117, 143003 (2016).

[40] U. Delić, M. Reisenbauer, K. Dare, D. Grass, V. Vuletić, N. Kiesel, and M. Aspelmeyer, Cooling of a levitated nanoparticle to the motional quantum ground state, Science 367, 892 (2020).

[41] W. P. Bowen and G. J. Milburn, Quantum Optomechanics (CRC Press, Boca Raton, Florida, 2015).

[42] A. Cavalleri, G. Ciani, R. Dolesi, A. Heptonstall, M. Hueller, D. Nicolodi, S. Rowan, D. Tombolato, S. Vitale, P. J. Wass et al., Increased Brownian Force Noise from Molecular Impacts in a Constrained Volume, Phys. Rev. Lett. 103, 140601 (2009).

[43] T. Seberson and F. Robicheaux, Distribution of laser shot-noise energy delivered to a levitated nanoparticle, Phys. Rev. A 102, 033505 (2020).

[44] ZARM Drop Tower Bremen User Manual (ZARM FABmbH, Bremen, Germany, 2008).

[45] H. Selig, H. Dittus, and C. Lämmerzahl, Drop tower microgravity improvement towards the nano-g level for the MICROSCOPE payload tests, Microgravity Sci. Technol. 22, 539 (2010).

[46] M. Armano, H. Audley, G. Auger, J. T. Baird, M. Bassan, P. Binetruy, M. Born, D. Bortoluzzi, N. Brandt, M. Caleno et al., Sub-Femto- $g$ Free Fall for Space-Based Gravitational Wave Observatories: LISA Pathfinder Results, Phys. Rev. Lett. 116, 231101 (2016).

[47] P. R. Saulson, Terrestrial gravitational noise on a gravitational wave antenna, Phys. Rev. D 30, 732 (1984)

[48] S. A. Hughes and K. S. Thorne, Seismic gravity-gradient noise in interferometric gravitational-wave detectors, Phys. Rev. D 58, 122002 (1998).

[49] K. S. Thorne and C. J. Winstein, Human gravity-gradient noise in interferometric gravitational-wave detectors, Phys. Rev. D 60, 082001 (1999).

[50] J. Harms, Terrestrial gravity fluctuations, Living Rev. Relativity 22, 6 (2019).

[51] M. Visser, Post-Newtonian particle physics in curved spacetime, arXiv:1802.00651.

[52] L. H. Ford, Casimir force between a dielectric sphere and a wall: A model for amplification of vacuum fluctuations, Phys. Rev. A 58, 4279 (1998).

[53] M. A. Schlosshauer, Decoherence and the Quantum-toClassical Transition (Springer, Berlin, Heidelberg, Germany, 2007).

[54] O. Romero-Isart, Quantum superposition of massive objects and collapse models, Phys. Rev. A 84, 052121 (2011).

[55] S. Kulas, C. Vogt, A. Resch, J. Hartwig, S. Ganske, J. Matthias, D. Schlippert, T. Wendrich, W. Ertmer, E. M. Rasel et al., Miniaturized lab system for future cold atom experiments in microgravity, Microgravity Sci. Technol. 29, 37 (2017).

[56] C. Brand, F. Kiałka, S. Troyer, C. Knobloch, K. Simonović, B. A. Stickler, K. Hornberger, and M. Arndt, Bragg Diffraction of Large Organic Molecules, Phys. Rev. Lett. 125, 033604 (2020). 